\title{
EFFECTS OF FRUSTRATED SURFACE IN HEISENBERG THIN FILMS
}

\author{
V. Thanh Ngo ${ }^{a, b}$ and H. T. Diep ${ }^{a *}$ \\ a Laboratoire de Physique Théorique et Modélisation, CNRS-Université de Cergy-Pontoise, UMR 8089 \\ 2, Avenue Adolphe Chauvin, 95302 Cergy-Pontoise Cedex, France \\ b Institute of Physics, P.O. Box 429 Bo Ho, Hanoi 10000, Vietnam
}

\begin{abstract}
We study by extensive Monte Carlo (MC) simulations and analytical Green function (GF) method effects of frustrated surfaces on the properties of thin films made of stacked triangular layers of atoms bearing Heisenberg spins with an Ising-like interaction anisotropy. We suppose that the in-plane surface interaction $J_{s}$ can be antiferromagnetic or ferromagnetic while all other interactions are ferromagnetic. We show that the ground-state spin configuration is non linear when $J_{s}$ is lower than a critical value $J_{s}^{c}$. The film surfaces are then frustrated. In the frustrated case, there are two phase transitions related to disorderings of surface and interior layers. There is a good agreement between MC and GF results. In addition, we show from MC histogram calculation that the value of the ratio of critical exponents $\gamma / \nu$ of the observed transitions is deviated from the values of two and three Ising universality classes. The origin of this deviation is discussed with general physical arguments.
\end{abstract}

PACS numbers:

\section{INTRODUCTION}

This paper deals with the effect of the frustration in magnetic thin films. The frustration is known to cause spectacular effects in various bulk spin systems. Its effects have been extensively studied during the last decade theoretically, experimentally and numerically. Frustrated model systems serve not only as testing ground for theories and approximations, but also to compare with experiments. $\frac{1}{1}$

On the other hand, surface physics and systems of nanoscales have been also enormously studied during the last twenty years. This is due in particular to applications in magnetic recording, let alone fundamental theoretical interests. Much is understood theoretically and experimentally in thin films where surfaces are 'clean' i.e. no impurities, no steps etc. 2,3,4,5,6.7 Less is known at least theoretically on complicated thin films with special surface conditions such as defects $\stackrel{8,9}{ }$, arrays of dots and magnetization reversal phenomenon. $10,11,12,13,14,15,16$

In this paper we study the frustration effect on properties of thin films made of stacked triangular lattices. In-plane interaction of the surfaces is antiferromagnetic and that of interior layers is ferromagnetic. The film surfaces are frustrated.

The paper is organized as follows. Section II is devoted to the description of our model. The ground state in the case of classical spins is determined as a function of the surface interaction. In section III, we consider the case of quantum spins and we apply the Green function technique to determine the layer magnetizations and the transition temperature as a function of the surface interaction. The classical ground state determined in section II is used here as starting (input)configuration for

\footnotetext{
${ }^{*}$ Corresponding author, E-mail: diep@u-cergy.fr
}

quantum spins. We are interested here in the effect of magnetic frustration on magnetic properties of thin films. A phase diagram is established showing interesting surface behaviors. Results from Monte Carlo simulations for classical spins are shown in section IV and compared to those obtained by the Green function method. We also calculate by Monte Carlo histogram technique the critical behavior of the phase transition observed here. Concluding remarks are given in Section V.

\section{MODEL}

It is known that many well-established theories failed to deal with frustrated spin systems. $\frac{1}{1}$ Among known striking effects due to frustration, let us mention the high ground-state (GS) degeneracy associated often with new symmetries which give rise sometimes to new kinds of phase transition. One of the systems which are most studied is the antiferromagnetic triangular lattice. Due to its geometry, the spins are frustrated under nearestneighbor $(\mathrm{NN})$ antiferromagnetic interaction. In the case of Heisenberg model, the frustration results in a $120^{\circ}$ GS structure: the NN spins form a $120^{\circ}$ angle alternately in the clockwise and counter-clockwise senses which are called left and right chiralities.

\section{A. Hamiltonian}

In this paper we consider a thin film made up by stacking $N_{z}$ planes of triangular lattice of $L \times L$ lattice sites.

The Hamiltonian is given by

$$
\mathcal{H}=-\sum_{\langle i, j\rangle} J_{i, j} \mathbf{S}_{i} \cdot \mathbf{S}_{j}-\sum_{<i, j>} I_{i, j} S_{i}^{z} S_{j}^{z}
$$

where $\mathbf{S}_{i}$ is the Heisenberg spin at the lattice site $i, \sum_{\langle i, j\rangle}$ indicates the sum over the NN spin pairs $\mathbf{S}_{i}$ and $\mathbf{S}_{j}$. The 
last term, which will be taken to be very small, is needed to make the film with a finite thickness to have a phase transition at a finite temperature in the case where all exchange interactions $J_{i, j}$ are ferromagnetic. This guarantees the existence of a phase transition at finite temperature, since it is known that a strictly two-dimensional system with an isotropic non-Ising spin model (XY or Heisenberg model) does not have long-range ordering at finite temperature $\underline{17}$

Interaction between two NN surface spins is equal to $J_{s}$. Interaction between layers and interaction between $\mathrm{NN}$ in interior layers are supposed to be ferromagnetic and all equal to $J=1$ for simplicity. The two surfaces of the film are frustrated if $J_{s}$ is antiferromagnetic $\left(J_{s}<0\right)$.

\section{B. Ground state}

In this paragraph, we suppose that the spins are classical. The classical ground state (GS) can be easily determined as shown below. Note that for antiferromagnetic systems, even for bulk materials, the quantum GS cannot be exactly determined. The classical Neel state is often used as starting configuration for quantum spins. We will follow the same line hereafter.

For $J_{s}>0$ (ferromagnetic interaction), the magnetic GS is ferromagnetic. However, when $J_{s}$ is negative the surface spins are frustrated. Therefore, there is a competition between the non collinear surface ordering and the ferromagnetic ordering due to the ferromagnetic interaction from the spins of the beneath layer.

We first determine the GS configuration for $I=I_{s}=$ 0.1 by using the steepest descent method : starting from a random spin configuration, we calculate the magnetic local field at each site and align the spin of the site in its local field. In doing so for all spins and repeat until the convergence is reached, we obtain in general the GS configuration, without metastable states in the present model. The result shows that when $J_{s}$ is smaller than a critical value $J_{s}^{c}$ the magnetic GS is obtained from the planar $120^{\circ}$ spin structure, supposed to be in the $X Y$ plane, by pulling them out of the spin $X Y$ plane by an angle $\beta$. The three spins on a triangle on the surface form thus an 'umbrella' with an angle $\alpha$ between them and an angle $\beta$ between a surface spin and its beneath neighbor (see Fig. 1). This non planar structure is due to the interaction of the spins on the beneath layer, just like an external applied field in the $z$ direction. Of course, when $J_{s}$ is larger than $J_{s}^{c}$ one has the collinear ferromagnetic GS as expected: the frustration is not strong enough to resist the ferromagnetic interaction from the beneath layer.

We show in Fig. $2 \cos (\alpha)$ and $\cos (\beta)$ as functions of $J_{s}$. The critical value $J_{s}^{c}$ is found between -0.18 and -0.19 . This value can be calculated analytically by assuming the 'umbrella structure'. For GS analysis, it suffices to consider just a cell shown in Fig 1, This is justified by the numerical determination discussed above. Further-

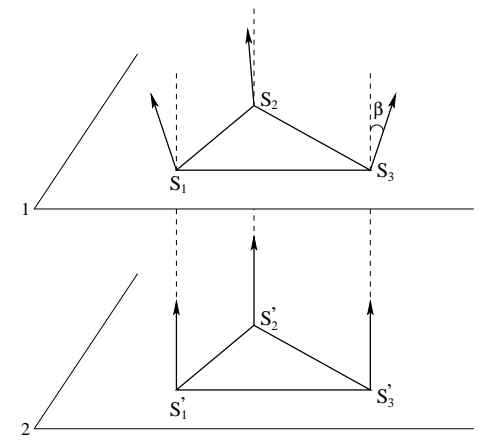

FIG. 1: Non collinear surface spin configuration. Angles between spins on layer 1 are all equal (noted $\alpha$ ), while angles between vertical spins are $\beta$.

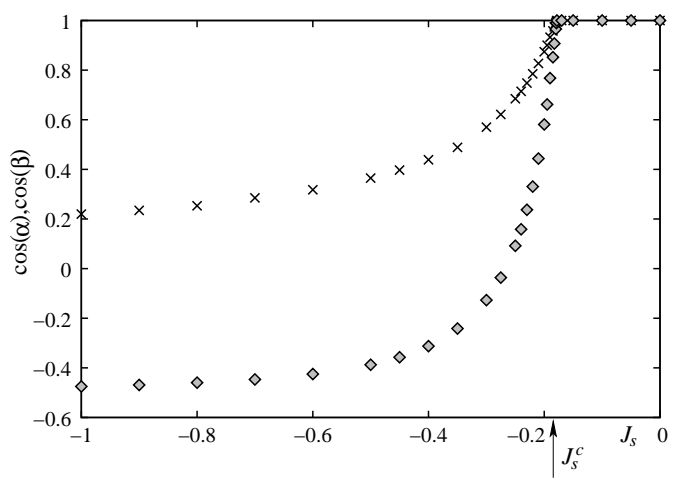

FIG. 2: $\cos (\alpha)$ (diamonds) and $\cos (\beta)$ (crosses) as functions of $J_{s}$. Critical value of $J_{s}^{c}$ is shown by the arrow.

more, we consider as a single solution all configurations obtained from each other by any global spin rotation.

Let us consider the full Hamiltonian (1). For simplicity, the interaction inside the surface layer is set equal $J_{s}$ $\left(-1 \leq J_{s} \leq 1\right)$ and all others are set equal to $J>0$. Also, we suppose that $I_{i, j}=I_{s}$ for spins on the surfaces with the same sign as $J_{s}$ and all other $I_{i, j}$ are equal to $I>0$ for the inside spins including interaction between a surface spin and the spin on the beneath layer.

The spins are numbered as in Fig. 11 $S_{1}, S_{2}$ and $S_{3}$ are the spins in the surface layer (first layer), $S_{1}^{\prime}, S_{2}^{\prime}$ and $S_{3}^{\prime}$ are the spins in the internal layer (second layer). The Hamiltonian for the cell is written as

$$
\begin{aligned}
H_{p}= & -6\left[J_{s}\left(\mathbf{S}_{1} \cdot \mathbf{S}_{2}+\mathbf{S}_{2} \cdot \mathbf{S}_{3}+\mathbf{S}_{3} \cdot \mathbf{S}_{1}\right)\right. \\
& +I_{s}\left(S_{1}^{z} S_{2}^{z}+S_{2}^{z} S_{3}^{z}+S_{3}^{z} S_{1}^{z}\right) \\
+ & J\left(\mathbf{S}_{1}^{\prime} \cdot \mathbf{S}_{2}^{\prime}+\mathbf{S}_{2}^{\prime} \cdot \mathbf{S}_{3}^{\prime}+\mathbf{S}_{3}^{\prime} \cdot \mathbf{S}_{1}^{\prime}\right) \\
& \left.+I\left(S_{1}^{\prime z} S_{2}^{\prime z}+S_{2}^{\prime z} S_{3}^{\prime z}+S_{3}^{\prime z} S_{1}^{\prime z}\right)\right] \\
- & 2 J\left(\mathbf{S}_{1} \cdot \mathbf{S}_{1}^{\prime}+\mathbf{S}_{2} \cdot \mathbf{S}_{2}^{\prime}+\mathbf{S}_{3} \cdot \mathbf{S}_{3}^{\prime}\right) \\
& -2 I\left(S_{1}^{z} S_{1}^{\prime z}+S_{2}^{\prime z} S_{2}^{\prime z}+S_{3}^{z} S_{3}^{\prime z}\right)
\end{aligned}
$$

Let us decompose each spin into two components: an $x y$ component, which is a vector, and a $z$ component $\mathbf{S}_{i}=\left(\mathbf{S}_{i}^{\|}, S_{i}^{z}\right)$. Only surface spins have $x y$ vector components. The angle between these $x y$ components of NN 
surface spins is $\gamma_{i, j}$ which is chosen by $\left(\gamma_{i, j}\right.$ is in fact the projection of $\alpha$ defined above on the $x y$ plane)

$$
\gamma_{1,2}=0, \gamma_{2,3}=\frac{2 \pi}{3}, \gamma_{3,1}=\frac{4 \pi}{3} .
$$

The angles $\beta_{i}$ and $\beta_{i}^{\prime}$ of the spin $\mathbf{S}_{i}$ and $\mathbf{S}_{i}^{\prime}$ with the $z$ axis are by symmetry

$$
\left\{\begin{array}{l}
\beta_{1}=\beta_{2}=\beta_{3}=\beta \\
\beta_{1}^{\prime}=\beta_{2}^{\prime}=\beta_{3}^{\prime}=0
\end{array}\right.
$$

The total energy of the cell (2), with $S_{i}=S_{i}^{\prime}=\frac{1}{2}$, can be rewritten as

$$
\begin{aligned}
H_{p} & =-\frac{9(J+I)}{2}-\frac{3(J+I)}{2} \cos \beta-\frac{9\left(J_{s}+I_{s}\right)}{2} \cos ^{2} \beta \\
& +\frac{9 J_{s}}{4} \sin ^{2} \beta .
\end{aligned}
$$

By a variational method, the minimum of the cell energy corresponds to

$$
\frac{\partial H_{p}}{\partial \beta}=\left(\frac{27}{2} J_{s}+9 I_{s}\right) \cos \beta \sin \beta+\frac{3}{2}(J+I) \sin \beta=0
$$

We have

$$
\cos \beta=-\frac{J+I}{9 J_{s}+6 I_{s}}
$$

For given values of $I_{s}$ and $I$, we see that the solution (6) exists for $J_{s} \leq J_{s}^{c}$ where the critical value $J_{s}^{c}$ is determined by $-1 \leq \cos \beta \leq 1$. For $I=-I_{s}=0.1$, $J_{s}^{c} \approx-0.1889 J$ in excellent agreement with the numerical result.

The classical GS determined here will be used as input GS configuration for quantum spins considered in the next section.

\section{GREEN FUNCTION METHOD}

Let us consider the quantum spin case. For a given value of $J_{s}$, we shall use the Green function method to calculate the layer magnetizations as functions of temperature. The details of the method in the case of non collinear spin configuration have been given in Ref. ${ }^{18}$. We briefly recall it here and show the application to the present model.

\section{A. Formalism}

We can rewrite the full Hamiltonian (1) in the local framework of the classical GS configuration as

$$
\mathcal{H}=-\sum_{<i, j>} J_{i, j}\left\{\frac{1}{4}\left(\cos \theta_{i j}-1\right)\left(S_{i}^{+} S_{j}^{+}+S_{i}^{-} S_{j}^{-}\right)\right.
$$

$$
\begin{aligned}
& +\frac{1}{4}\left(\cos \theta_{i j}+1\right)\left(S_{i}^{+} S_{j}^{-}+S_{i}^{-} S_{j}^{+}\right) \\
& +\frac{1}{2} \sin \theta_{i j}\left(S_{i}^{+}+S_{i}^{-}\right) S_{j}^{z}-\frac{1}{2} \sin \theta_{i j} S_{i}^{z}\left(S_{j}^{+}+S_{j}^{-}\right) \\
& \left.+\cos \theta_{i j} S_{i}^{z} S_{j}^{z}\right\}-\sum_{<i, j>} I_{i, j} S_{i}^{z} S_{j}^{z}
\end{aligned}
$$

where $\cos \left(\theta_{i j}\right)$ is the angle between two NN spins determined classically in the previous section.

Following Tahir-Kheli and ter Haar, ${ }^{19}$ we define two double-time Green functions by

$$
\begin{aligned}
G_{i j}\left(t, t^{\prime}\right) & =\ll S_{i}^{+}(t) ; S_{j}^{-}\left(t^{\prime}\right) \gg, \\
F_{i j}\left(t, t^{\prime}\right) & =\ll S_{i}^{-}(t) ; S_{j}^{+}\left(t^{\prime}\right) \gg .
\end{aligned}
$$

The equations of motion for $G_{i j}\left(t, t^{\prime}\right)$ and $F_{i j}\left(t, t^{\prime}\right)$ read

$$
\begin{aligned}
i \frac{d}{d t} G_{i, j}\left(t, t^{\prime}\right) & =\left\langle\left[S_{i}^{+}(t), S_{j}^{-}\left(t^{\prime}\right)\right]\right\rangle \delta\left(t-t^{\prime}\right) \\
& -\left\langle\left\langle\left[\mathcal{H}, S_{i}^{+}(t)\right] ; S_{j}^{-}\left(t^{\prime}\right)\right\rangle\right\rangle, \\
i \frac{d}{d t} F_{i, j}\left(t, t^{\prime}\right) & =\left\langle\left[S_{i}^{-}(t), S_{j}^{-}\left(t^{\prime}\right)\right]\right\rangle \delta\left(t-t^{\prime}\right) \\
& -\left\langle\left\langle\left[\mathcal{H}, S_{i}^{-}(t)\right] ; S_{j}^{-}\left(t^{\prime}\right)\right\rangle\right\rangle,
\end{aligned}
$$

We neglect higher order correlations by using the Tyablikov decoupling scheme ${ }^{20}$ which is known to be valid for exchange terms. ${ }^{21}$ Then, we introduce the Fourier transforms

$$
\begin{gathered}
G_{i, j}\left(t, t^{\prime}\right)=\frac{1}{\Delta} \iint d \mathbf{k}_{x y} \frac{1}{2 \pi} \int_{-\infty}^{+\infty} d \omega e^{-i \omega\left(t-t^{\prime}\right)} \\
g_{n, n^{\prime}}\left(\omega, \mathbf{k}_{x y}\right) e^{i \mathbf{k}_{x y} \cdot\left(\mathbf{R}_{i}-\mathbf{R}_{j}\right)} \\
F_{i, j}\left(t, t^{\prime}\right)=\frac{1}{\Delta} \iint d \mathbf{k}_{x y} \frac{1}{2 \pi} \int_{-\infty}^{+\infty} d \omega e^{-i \omega\left(t-t^{\prime}\right)} \\
f_{n, n^{\prime}}\left(\omega, \mathbf{k}_{x y}\right) e^{i \mathbf{k}_{x y} \cdot\left(\mathbf{R}_{i}-\mathbf{R}_{j}\right)}
\end{gathered}
$$

where $\omega$ is the spin-wave frequency, $\mathbf{k}_{x y}$ denotes the wavevector parallel to $x y$ planes, $\mathbf{R}_{i}$ is the position of the spin at the site $i, n$ and $n^{\prime}$ are respectively the index of the layers where the sites $i$ and $j$ belong to. The integral over $\mathbf{k}_{x y}$ is performed in the first Brillouin zone whose surface is $\Delta$ in the $x y$ reciprocal plane.

The Fourier transforms of the retarded Green functions satisfy a set of equations rewritten under a matrix form

$$
\mathbf{M}(\omega) \mathbf{g}=\mathbf{u}
$$

where $\mathbf{M}(\omega)$ is a square matrix $\left(2 N_{z} \times 2 N_{z}\right), \mathbf{g}$ and $\mathbf{u}$ are the column matrices which are defined as follows

$$
\mathbf{g}=\left(\begin{array}{c}
g_{1, n^{\prime}} \\
f_{1, n^{\prime}} \\
\vdots \\
g_{N_{z}, n^{\prime}} \\
f_{N_{z}, n^{\prime}}
\end{array}\right), \quad \mathbf{u}=\left(\begin{array}{c}
2\left\langle S_{1}^{z}\right\rangle \delta_{1, n^{\prime}} \\
0 \\
\vdots \\
2\left\langle S_{N_{z}}^{z}\right\rangle \delta_{N_{z}, n^{\prime}} \\
0
\end{array}\right)
$$


and

$$
\mathbf{M}(\omega)=\left(\begin{array}{ccccc}
A_{1}^{+} & B_{1} & D_{1}^{+} & D_{1}^{-} & \cdots \\
-B_{1} & A_{1}^{-} & -D_{1}^{-} & -D_{1}^{+} & \vdots \\
\vdots & \ldots & \ldots & \cdots & \vdots \\
\vdots & C_{N_{z}}^{+} & C_{N_{z}}^{-} & A_{N_{z}}^{+} & B_{N_{z}} \\
\cdots & -C_{N_{z}}^{-} & -C_{N_{z}} & -B_{N_{z}} & A_{N_{z}}^{-}
\end{array}\right),
$$

where

$$
\begin{aligned}
A_{n}^{ \pm}= & \omega \pm\left[\frac{1}{2} J_{n}\left\langle S_{n}^{z}\right\rangle(Z \gamma)\left(\cos \theta_{n}+1\right)\right. \\
- & J_{n}\left\langle S_{n}^{z}\right\rangle Z \cos \theta_{n}-J_{n, n+1}\left\langle S_{n+1}^{z}\right\rangle \cos \theta_{n, n+1} \\
- & J_{n, n-1}\left\langle S_{n-1}^{z}\right\rangle \cos \theta_{n, n-1}-Z I_{n}\left\langle S_{n}^{z}\right\rangle \\
& \left.-I_{n, n+1}\left\langle S_{n+1}^{z}\right\rangle-I_{n, n-1}\left\langle S_{n-1}^{z}\right\rangle\right] \\
B_{n}= & \frac{1}{2} J_{n}\left\langle S_{n}^{z}\right\rangle\left(\cos \theta_{n}-1\right)(Z \gamma) \\
C_{n}^{ \pm}= & \frac{1}{2} J_{n, n-1}\left\langle S_{n}^{z}\right\rangle\left(\cos \theta_{n, n-1} \pm 1\right) \\
D_{n}^{ \pm}= & \frac{1}{2} J_{n, n+1}\left\langle S_{n}^{z}\right\rangle\left(\cos \theta_{n, n+1} \pm 1\right)
\end{aligned}
$$

in which, $Z=6$ is the number of in-plane NN, $\theta_{n, n \pm 1}$ the angle between two NN spins belonging to the layers $n$ and $n \pm 1, \theta_{n}$ the angle between two in-plane $\mathrm{NN}$ in the layer $n$, and

$$
\gamma=\left[2 \cos \left(k_{x} a\right)+4 \cos \left(k_{y} a / 2\right) \cos \left(k_{y} a \sqrt{3} / 2\right)\right] / Z .
$$

Here, for compactness we have used the following notations:

i) $J_{n}$ and $I_{n}$ are the in-plane interactions. In the present model $J_{n}$ is equal to $J_{s}$ for the two surface layers and equal to $J$ for the interior layers. All $I_{n}$ are set to be $I$.

ii) $J_{n, n \pm 1}$ and $I_{n, n \pm 1}$ are the interactions between a spin in the $n^{\text {th }}$ layer and its neighbor in the $(n \pm 1)^{t h}$ layer. Of course, $J_{n, n-1}=I_{n, n-1}=0$ if $n=1, J_{n, n+1}=$ $I_{n, n+1}=0$ if $n=N_{z}$.

Solving $\operatorname{det}|\mathbf{M}|=0$, we obtain the spin-wave spectrum $\omega$ of the present system. The solution for the Green function $g_{n, n}$ is given by

$$
g_{n, n}=\frac{|\mathbf{M}|_{n}}{|\mathbf{M}|},
$$

where $|\mathbf{M}|_{n}$ is the determinant made by replacing the $n$-th column of $|\mathbf{M}|$ by $\mathbf{u}$ in (15). Writing now

$$
|\mathbf{M}|=\prod_{i}\left(\omega-\omega_{i}\left(\mathbf{k}_{x y}\right)\right)
$$

one sees that $\omega_{i}\left(\mathbf{k}_{x y}\right), i=1, \cdots, N_{z}$, are poles of the Green function $g_{n, n} . \omega_{i}\left(\mathbf{k}_{x y}\right)$ can be obtained by solving $|\mathbf{M}|=0$. In this case, $g_{n, n}$ can be expressed as

$$
g_{n, n}=\sum_{i} \frac{h_{n}\left(\omega_{i}\left(\mathbf{k}_{x y}\right)\right)}{\left(\omega-\omega_{i}\left(\mathbf{k}_{x y}\right)\right)},
$$

where $h_{n}\left(\omega_{i}\left(\mathbf{k}_{x y}\right)\right)$ is

$$
h_{n}\left(\omega_{i}\left(\mathbf{k}_{x y}\right)\right)=\frac{|\mathbf{M}|_{n}\left(\omega_{i}\left(\mathbf{k}_{x y}\right)\right)}{\prod_{j \neq i}\left(\omega_{j}\left(\mathbf{k}_{x y}\right)-\omega_{i}\left(\mathbf{k}_{x y}\right)\right)} .
$$

Next, using the spectral theorem which relates the correlation function $\left\langle S_{i}^{-} S_{j}^{+}\right\rangle$to the Green functions, $\stackrel{22}{,}$ one has

$$
\begin{aligned}
\left\langle S_{i}^{-} S_{j}^{+}\right\rangle & =\lim _{\varepsilon \rightarrow 0} \frac{1}{\Delta} \iint d \mathbf{k}_{x y} \int_{-\infty}^{+\infty} \frac{i}{2 \pi}\left(g_{n, n^{\prime}}(\omega+i \varepsilon)\right. \\
& \left.-g_{n, n^{\prime}}(\omega-i \varepsilon)\right) \frac{d \omega}{e^{\beta \omega}-1} e^{i \mathbf{k}_{x y} \cdot\left(\mathbf{R}_{i}-\mathbf{R}_{j}\right)},
\end{aligned}
$$

where $\epsilon$ is an infinitesimal positive constant and $\beta=$ $1 / k_{B} T, k_{B}$ being the Boltzmann constant. For spin $S=$ $1 / 2$, the thermal average of the $z$ component of the $i-t h$ spin belonging to the $n-t h$ layer is given by

$$
\left\langle S_{i}^{z}\right\rangle=\frac{1}{2}-\left\langle S_{i}^{-} S_{i}^{+}\right\rangle
$$

In the following we shall use the case of spin one-half. Note that for the case of general $S$, the expression for $\left\langle S_{i}^{z}\right\rangle$ is more complicated since it involves higher quantities such as $\left\langle\left(S_{i}^{z}\right)^{2}\right\rangle$.

Using the Green function presented above, we can calculate self-consistently various physical quantities as functions of temperature $T$. The first important quantity is the temperature dependence of the angle between each spin pair. This can be calculated in a self-consistent manner at any temperature by minimizing the free energy at each temperature to get the correct value of the angle as it has been done for a frustrated bulk spin systems ${ }^{23}$ In this paper, we limit ourselves to the self-consistent calculation of the layer magnetizations which allows us to establish the phase diagram as seen in the following.

For numerical calculation, we used $I=0.1 J$ with $J=$ 1. For positive $J_{s}$, we take $I_{s}=0.1$ and for negative $J_{s}$, we use $I_{s}=-0.1$. A size of $80^{2}$ points in the first Brillouin zone is used for numerical integration. We start the self-consistent calculation from $T=0$ with a small step for temperature $5 \times 10^{-3}$ or $10^{-1}$ (in units of $J / k_{B}$ ). The convergence precision has been fixed at the fourth figure of the values obtained for the layer magnetizations.

\section{B. Phase transition and phase diagram of the quantum case}

We first show an example where $J_{s}=-0.5$ in Fig. 3 , As seen, the surface-layer magnetization is much smaller than the second-layer one. In addition there is a strong spin contraction at $T=0$ for the surface layer. This is due to the antiferromagnetic nature of the in-plane surface interaction $J_{s}$. One sees that the surface becomes disordered at a temperature $T_{1} \simeq 0.2557$ while the second layer remains ordered up to $T_{2} \simeq 1.522$. Therefore, 
the system is partially disordered for temperatures between $T_{1}$ and $T_{2}$. This result is very interesting because it confirms again the existence of the partial disorder in quantum spin systems observed earlier in bulk frustrated quantum spin systems $\frac{18,23}{}$ Note that between $T_{1}$ and $T_{2}$, the ordering of the second layer acts as an external field on the first layer, inducing therefore a small value of its magnetization. A further evidence of the existence of the surface transition will be provided with the surface susceptibility in the MC results shown below.

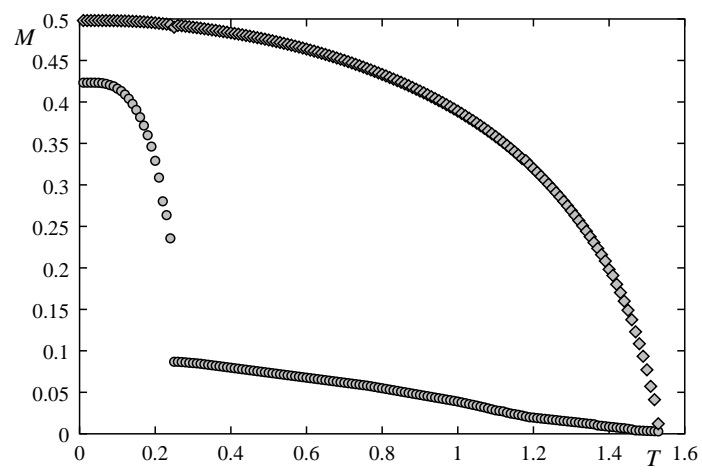

FIG. 3: First two layer-magnetizations obtained by the Green function technique vs. $T$ for $J_{s}=-0.5$ with $I=-I_{s}=0.1$. The surface-layer magnetization (lower curve) is much smaller than the second-layer one. See text for comments.

Figure 4 4 shows the non frustrated case where $J_{s}=0.5$, with $I=I_{s}=0.1$. As seen, the first-layer magnetization is smaller than the second-layer one. There is only one transition temperature. Note the difficulty for numerical convergency when the magnetizations come close to zero.

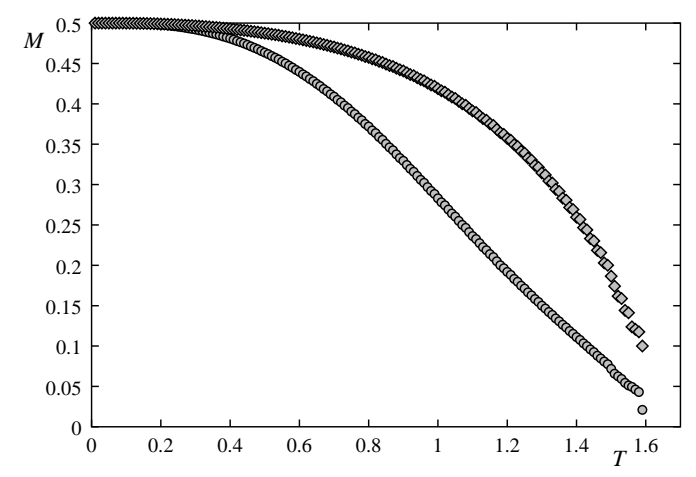

FIG. 4: First two layer-magnetizations obtained by the Green function technique vs. $T$ for $J_{s}=0.5$ with $I=I_{s}=0.1$.

We show in Fig. 5 the phase diagram in the space $\left(J_{s}, T\right)$. Phase I denotes the ordered phase with surface non collinear spin configuration, phase II indicates the collinear ordered state, and phase III is the paramagnetic phase. Note that the surface transition does not exist for $J_{s} \geq J_{s}^{c}$.

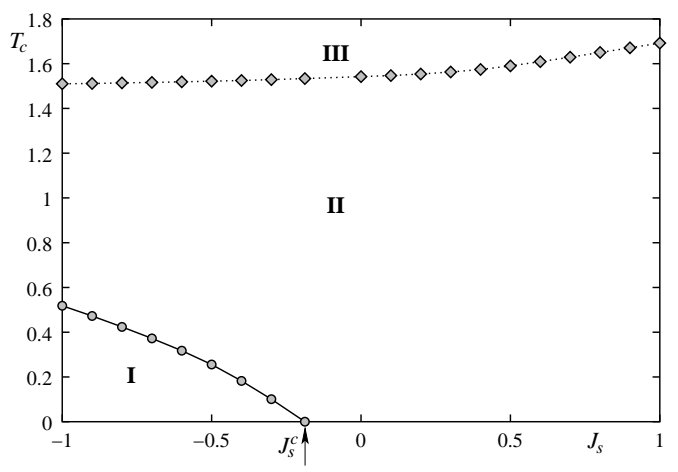

FIG. 5: Phase diagram in the space $\left(J_{s}, T\right)$ for the quantum Heisenberg model with $N_{z}=4, I=\left|I_{s}\right|=0.1$. See text for the description of phases I to III.

\section{MONTE CARLO RESULTS}

It is known that methods for quantum spins, such as spin-wave theory or Green function method presented above, suffer at high temperatures. Spin-wave theory, even with magnon-magnon interactions taken into account, cannot go to temperatures close to $T_{c}$. Green function method on the other hand can go up to $T_{c}$ but due to the decoupling scheme, it cannot give correct critical behavior at $T_{c}$. Fortunately, we know that quantum spin systems behave as their classical counterparts at high $T$. So, to see if the phase diagram obtained in the previous section for the quantum model is precise or not, we can consider its classical version and use MC simulations to obtain the phase diagram for comparison. MC simulations are excellent means to overcome approximations used in analytic calculations for the high $T$ region as discussed above.

In this paragraph, we show the results obtained by $\mathrm{MC}$ simulations with the Hamiltonian (11) but the spins are the classical Heisenberg model of magnitude $S=1$.

The film sizes are $L \times L \times N_{z}$ where $N_{z}=4$ is the number of layers (film thickness) taken as in the quantum case presented above. We use here $L=24,36,48,60$ to study finite-size effects as shown below. Periodic boundary conditions are used in the $X Y$ planes. The equilibrating time is about $10^{6} \mathrm{MC}$ steps per spin and the averaging time is $2 \times 10^{6} \mathrm{MC}$ steps per spin. $J=1$ is taken as unit of energy in the following.

Let us show in Fig. 6 the layer magnetization of the first two layers as a function of $T$, in the case $J_{s}=0.5$ with $N_{z}=4$ (the third and fourth layers are symmetric). Though we observe a smaller magnetization for the surface layer, there is clearly no surface transition just as in the quantum case.

In Fig. 7 we show a frustrated case where $J_{s}=-0.5$. The surface layer in this case becomes disordered at a temperature much lower than that for the second layer. Note that the surface magnetization is not saturated to 1 at $T=0$. This is because the surface spins make an 


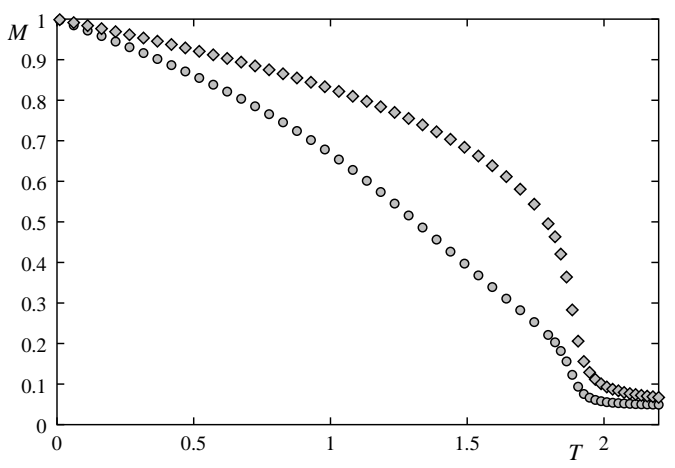

FIG. 6: Magnetizations of layer 1 (circles) and layer 2 (diamonds) versus temperature $T$ in unit of $J / k_{B}$ for $J_{s}=0.5$ with $I=I_{s}=0.1, L=36$.

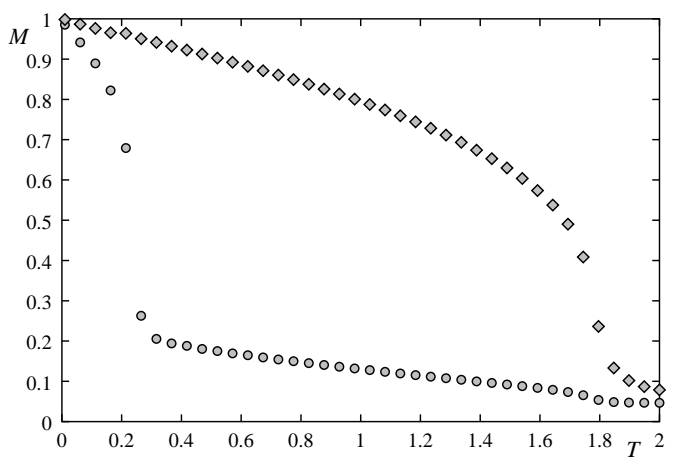

FIG. 7: Magnetizations of layer 1 (circles) and layer 2 (diamonds) versus temperature $T$ in unit of $J / k_{B}$ for $J_{s}=-0.5$ with $I=-I_{s}=0.1, L=36$.

angle with the $z$ axis so their $z$ component is less than 1 in the GS.

Figures 8 shows the susceptibilities of the first and second layers in the case where $J_{s}=0.5$ with $I=I_{s}=0.1$ where one observes the peaks at the same temperature indicating a single transition in contrast to the frustrated case shown in Fig. 9. These results confirm the above results of layer magnetizations.

To establish the phase diagram, the transition temperatures are taken at the change of curvature of the layer magnetizations, i.e. at the maxima of layer susceptibilities shown before. Figure 10 shows the phase diagram obtained in the space $\left(J_{s}, T\right)$. Interesting enough, this phase diagram resembles remarkably to that obtained for the quantum counterpart model shown in Fig. 5

Let us study the finite size effect of the phase transitions shown in Fig. 10. To this end we use the histogram technique which has been proved so far to be excellent for the calculation of critical exponents $\underline{24,25,26}$ The principle is as follows. Using the Metropolis algorithm to determine approximately the critical temperature region, then choosing a temperature $T_{0}$ as close as possible to the presupposed transition temperature. We then make a very long run at $T_{0}$ to establish an energy histogram. From

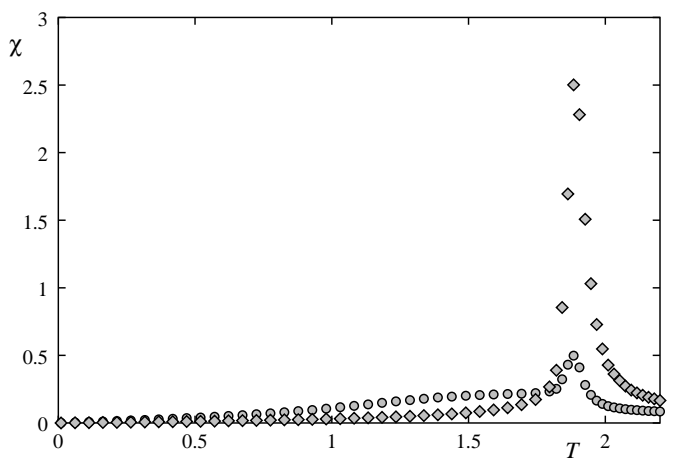

FIG. 8: Susceptibilities of layer 1 (circles) and layer 2 (diamonds) versus temperature $T$ in unit of $J / k_{B}$ for $J_{s}=0.5$ with $I=I_{s}=0.1, L=36$.

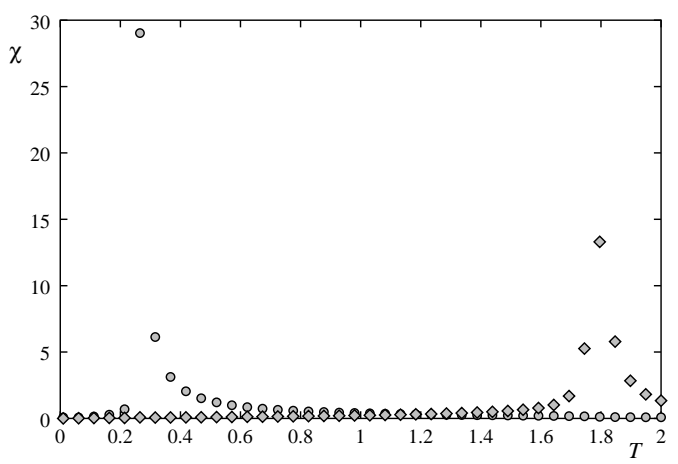

FIG. 9: Susceptibility of layer 1 (circles) and layer 2 (diamonds) versus temperature $T$ in unit of $J / k_{B}$ for $J_{s}=-0.5$ with $I=-I_{s}=0.1, L=36$. Note that for clarity, the susceptibility of the layer 2 has been multiplied by a factor 5 .

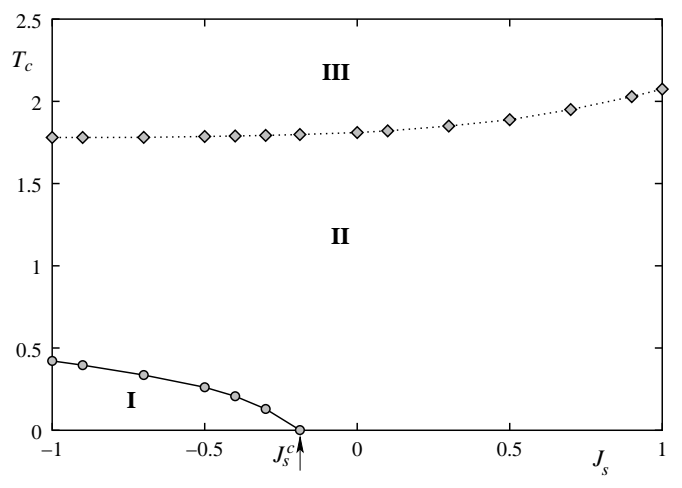

FIG. 10: Phase diagram in the space $\left(J_{s}, T\right)$ for the classical Heisenberg model with $N_{z}=4, I=\left|I_{s}\right|=0.1$. Phases I to III have the same meanings as those in Fig. 5 .

formulae established using the statistical canonical distribution, we can calculate physical quantities in a continuous manner for temperatures around $T_{0} \stackrel{24,25,26}{2}$ We do not have problem to identify the transition temperature as well as the maximal values of fluctuation quantities such as specific heat and susceptibility. 
Figure 11 shows the susceptibility versus $T$ for $L=$ $36,48,60$ in the case of $J_{s}=0.5$. For presentation convenience, the size $L=24$ has been removed since the peak for this case is rather flat in the scale of the figure. However, it shall be used to calculate the critical exponent $\gamma$ for the transition. As seen, the maximum $\chi^{\max }$ of the susceptibility increases with increasing $L$.

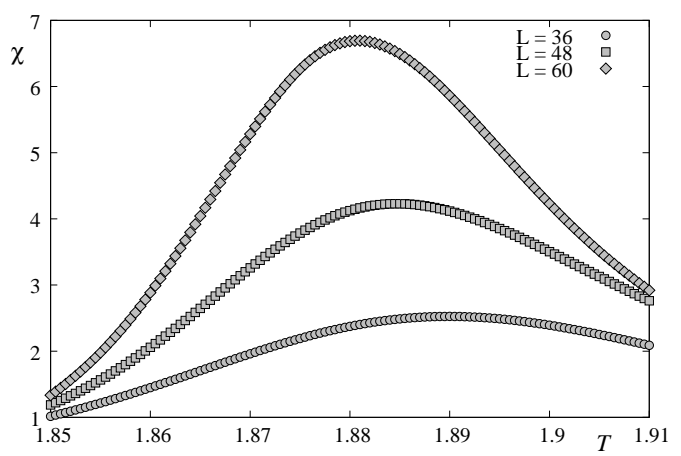

FIG. 11: Susceptibility versus $T$ for $L=36,48,60$ with $J_{s}=$ 0.5 and $I=I_{s}=0.1$.

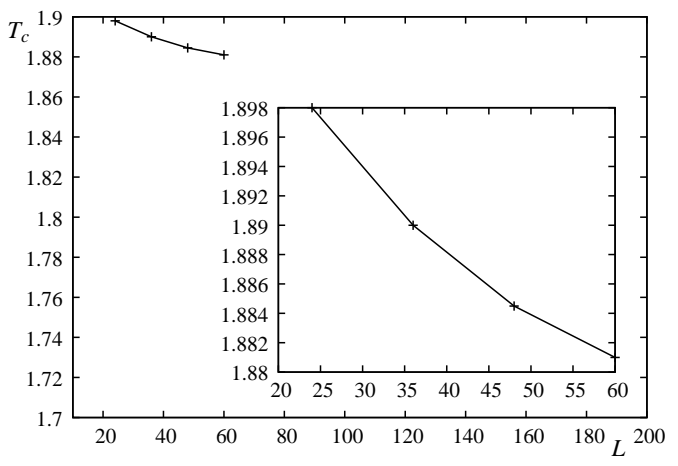

FIG. 12: Transition temperature versus $L$ with $J_{s}=0.5$ and $I=I_{s}=0.1$. The inset shows the enlarged scale.

For completeness, we show in Fig. 12 the transition temperature as a function of $L$. A rough extrapolation to infinity gives $T_{c}^{\infty} \simeq 1.86 \pm 0.02$.

In the frustrated case, i.e. $J_{s}<J_{s}^{c}$, we perform the same calculation for finite-size effect. Note that in this case there are two phase transitions. We show in Fig. 13 the layer susceptibilities as functions of $T$ for different $L$. As seen, both surface and second-layer transitions have a strong size dependence.

We show in Fig. 14 and 15 the size dependence of the transition temperatures $T_{1}$ (surface transition) and $T_{2}$ (second-layer transition).

The size dependence of the maxima observed above allows us to estimate the ratio $\gamma / \nu$. We show now $\ln \chi^{\max }$ as a function of $\ln L$ for the different cases studied above. Figure 16(a) and (b) correspond respectively to the transitions of surface and second layer occurring in the frustrated case with $J_{s}=-0.5$, while Figure 16(c) corre-
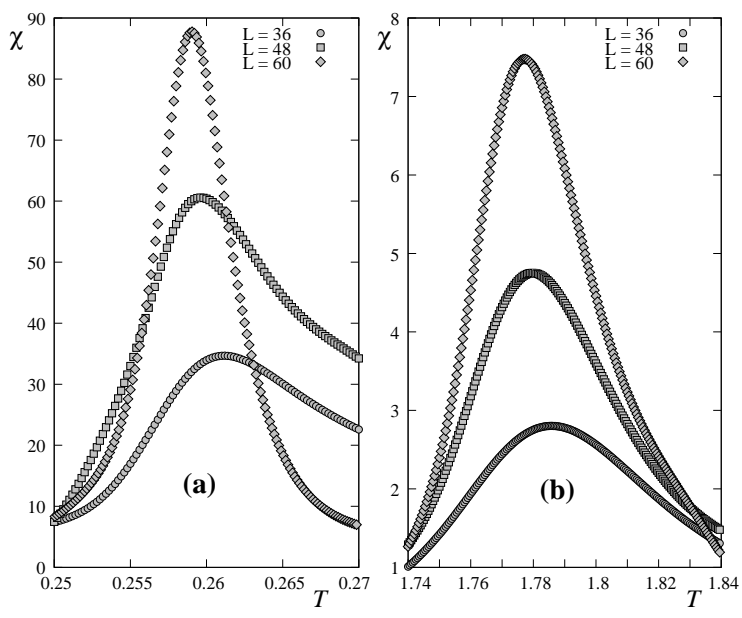

FIG. 13: Layer susceptibilities versus $T$ for $L=36,48,60$ with $J_{s}=-0.5$ and $I=-I_{s}=0.1$. Left (right) figure corresponds to the first (second) layer susceptibility.

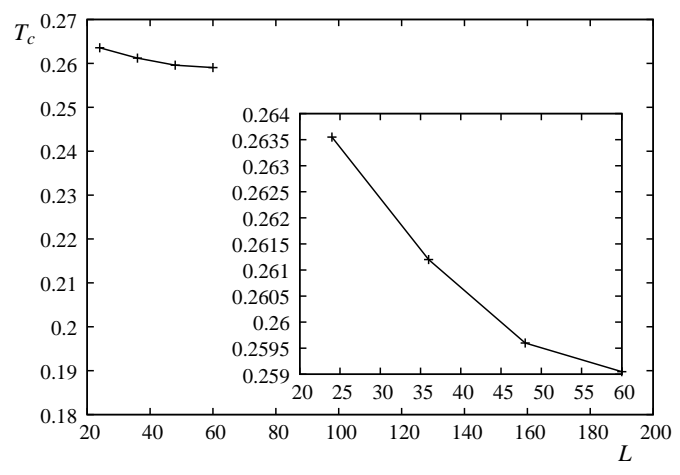

FIG. 14: Transition temperature versus $L$ for the surface layer in the case $J_{s}=-0.5$ with $I=-I_{s}=0.1$. The inset shows the enlarged scale.

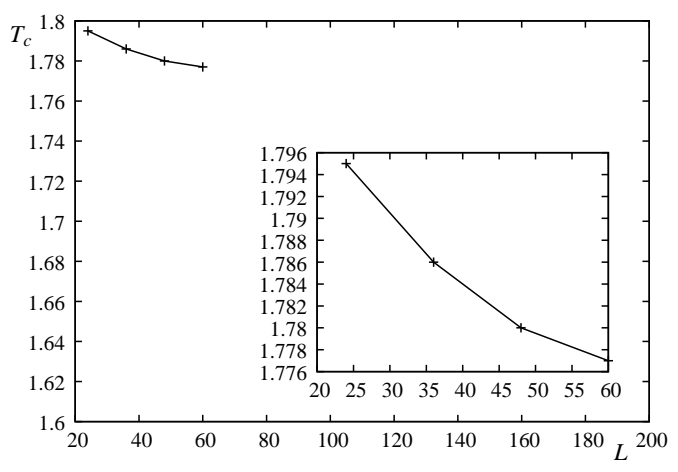

FIG. 15: Transition temperature versus $L$ for the second layer in the case $J_{s}=-0.5$ with $I=-I_{s}=0.1$. The inset shows the enlarged scale.

sponds to the unique transition occurring in the non frustrated case with $J_{s}=0.5$. The slopes of these straight lines give $\gamma / \nu \simeq 1.864 \pm 0.034$ (a), $1.878 \pm 0.027$ (b), $1.801 \pm 0.027(\mathrm{c})$. The errors were estimated from the 


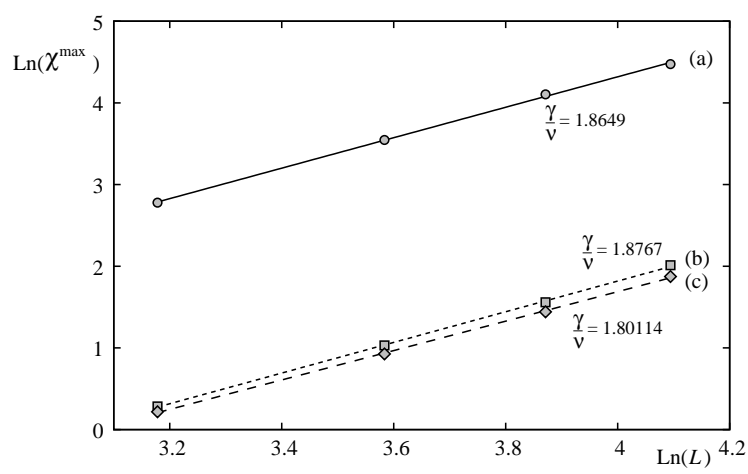

FIG. 16: Maximum of surface-layer susceptibility versus $L$ for $L=24,36,48,60$ with $J_{s}=-0.5(\mathrm{a}, \mathrm{b}), J_{s}=0.5$ (c) and $I=\left|I_{s}\right|=0.1$, in the $\ln -\ln$ scale. The slope gives $\gamma / \nu$ indicated in the figure for each case. See text for comments.

mean least-square fitting and errors on the peak values obtained with different values of $T_{0}$ (multi histogram technique). Within errors, the first two values, which correspond to the frustrated case, can be considered as identical, while the last one corresponding to the non frustrated case is different. We will return to this point later.

At this stage, we would like to emphasize the following points. First, we observe that these values of $\gamma / \nu$ are found to be between those of the two-dimensional (2D) Ising model $(\gamma / \nu=1.75)$ and the three-dimensional one $(\gamma / \nu=1.241 / 0.63 \simeq 1.97)$. A question which naturally arises on the role of the Ising-like anisotropy term, of the two-fold chiral symmetry and of the film thickness. The role of the anisotropy term and the chiral symmetry is obvious: the Ising character should be observed in the result (we return to this point below). It is however not clear for the effect of the thickness. Some arguments, such as those from renormalization group, say that the correlation length in the direction perpendicular to the film is finite, hence it is irrelevant to the criticality, the $2 \mathrm{D}$ character therefore should be theoretically preserved. We think that such arguments are not always true because it is difficult to conceive that when the film thickness becomes larger and larger the 2D universality should remain. Instead, we believe that that the finite thickness of the film affects the 2D universality in one way or another, giving rise to "effective" critical exponents with values deviated from the $2 \mathrm{D}$ ones. The larger the thickness is, the stronger this deviation becomes. The observed values of $\gamma / \nu$ shown above may contain an effect of a 2D-3D cross-over. At this point, we would like to emphasize that, in the case of simple surface conditions, i.e. no significant deviation of the surface parameters with respect to those of the bulk, the bulk behavior is observed when the thickness becomes larger than a dozen of atomic layers: $: \underline{\underline{5}}$ surface effects are insignificant on thermodynamic properties of the film. There are therefore reasons to believe that there should be a crossover from $2 \mathrm{D}$ to $3 \mathrm{D}$ at some film thickness. Of course, this is an important issue which needs to be theoretically clarified in the future. We return now to the effect of Ising anisotropy and chiral symmetry. The deviation from the $2 \mathrm{D}$ values may result in part from a complex coupling between the Ising symmetry, due to anisotropy and chirality, and the continuous nature of the classical Heisenberg spins studied here. This deviation may be important if the anisotropy constant $I$ is small. For the effect chiral symmetry, it is a complex matter. To show the complexity in determining the critical universality with chiral symmetry, let us discuss about a simpler case with similar chiral symmetry: the XY model on the fully frustrated Villain's lattice. There has been a large number of investigations on the nature of the transition observed in this $2 \mathrm{D}$ case in the context of the frustration effects. $\stackrel{1,27,28}{1 n}$ this model, though the chirality symmetry argument says that the transition should be of $2 \mathrm{D}$ Ising universality class, many investigators found a deviation of critical exponents from those of the $2 \mathrm{D}$ case (see review in Ref ${ }^{28}$ ). For example, the following values are found for the critical exponent $\nu: \nu=0.889^{29}$ and $\nu=0.813 .30$ These values are close to that obtained for the single transition in a mixed $X Y$-Ising model which is $0.85 \stackrel{31,32}{ }$ It is now believed that the XY character of the spins affects the Ising chiral symmetry giving rise to those deviated critical exponents. Similarly, in the case of thin film studied here, we do not deal with the discrete Ising model but rather an Ising-like Heisenberg model. The Ising character due to chiral symmetry of the transition at the surface is believed to be perturbed by the continuous nature of Heisenberg spins. The transition of interior layer shown in Fig. 16(b) suffers similar but not the same effects because of the absence of chiral symmetry on this layer. So the value $\gamma / \nu$ is a little different. In the non frustrated case shown in Fig. 16(c), the deviation from the 2D Ising universality class is less important because of the absence of the chiral symmetry. This small deviation is believed to stem mainly from the continuous nature of Heisenberg spins.

To conclude this paragraph, we believe, from physical arguments given above, that the deviation from 2D Ising universality class of the transitions observed here is due to, in an decreasing order of importance, the effect of the coupling between the continuous degree of freedom of Heisenberg spin to the chiral symmetry, the small Isinglike anisotropy and the film thickness.

\section{CONCLUDING REMARKS}

We have studied, by means of a Green function method and MC simulations, the Heisenberg spin model with an Ising-like interaction anisotropy in thin films of stacked triangular lattices. The two surfaces of the film are frustrated. We found that surface spin configuration is non collinear when surface antiferromagnetic interaction is smaller than a critical value $J_{s}^{c}$. In the non collinear regime, the surface layer is disordered at a temperature 
lower than that for interior layers ("soft" surface). This can explain the so-called "magnetically dead surface" observed in some materials. ${ }^{33.34}$ The surface transition disappears for $J_{s}$ larger than the critical value $J_{s}^{c}$. A phase diagram is established in the space $\left(T, J_{s}\right)$. A good agreement between the Green function method and the MC simulation is observed. This is due to the fact that at high temperatures where the transition takes place, the quantum nature of spins used in the GF is lost so that we should find results of classical spins used in MC simulations. We have also studied by MC histogram technique the critical behavior of the phase transition using the finite-size effects. The result of the ratio of critical exponents $\gamma / \nu$ shows that the nature of the transition is complicated due to the influence of several physical mech- anisms. The symmetry of the ground state alone cannot explain such a result. We have outlined a number of the most relevant mechanisms. Finally, we note that in surface magnetism the low surface magnetization experimentally observed ${ }^{33.34}$ has been generally attributed to the effects of the reduction of magnetic moments of surface atoms and/or the surface-localized low-lying magnon modes. The model considered in this paper adds another origin for the low surface magnetization: surface frustration. It completes the list of possible explanations for experimental observations in thin films.

One of us (NVT) thanks 'World Laboratory' for financial support. This work is supported by a contract between CNRS (France) and VAST (Vietnam).
1 See reviews on theories and experiments given in Frustrated Spin Systems, ed. H. T. Diep, World Scientific (2005).

2 K. Binder, in Phase Transitions and Critical Phenomena, ed. by C. Domb, J.L. Lebowitz (Academic, London, 1983) vol. 8.

3 H.W. Diehl, in Phase Transitions and Critical Phenomena, ed. by C. Domb, J.L. Lebowitz (Academic, London, 1986) vol. 10, H.W. Diehl, Int. J. Mod. Phys. B 11, 3503 (1997).

${ }^{4}$ H. T. Diep, J.C. S. Levy and O. Nagai, Phys. Stat. Solidi (b) 93, 351 (1979).

${ }^{5}$ H. T. Diep, Phys. Stat. Solidi (b) , 103, 809 (1981).

${ }^{6}$ H. T. Diep, Phys. Rev. B 43, 8509 (1991).

7 V. Thanh Ngo, H. Viet Nguyen, H. T. Diep and V. Lien Nguyen, Phys. Rev. B. 69, 134429 (2004).

8 M.-C. Chung et al, Eur. Phys. J. B 18655 (2000).

9 J. Shen and al. Phys. Rev. B 56, 11134 (1997).

10 F. Rousseau and al. J. Vac. Sci Technol. B 13, 2787 (1995).

11 L. Kong, L. Zhuang and S. Chu. IEEE Trans Magn 33, 3019 (1997).

12 V. Grolier, J. Ferrr, A. Maziewski, E. Stefanowicz and D. Renard. J. Appl. Phys. 73, 5939 (1993).

13 J. P Jamet and al. Phys. Rev. B 57, 14320 (1998).

14 P. Meyer and al. Phys. Rev. Lett. 81, 5656 (1998).

15 C. Santamaria and H. T. Diep, J. Magn. Magn. Mater. 212, 23 (2000).

16 C. Santamaria and H. T. Diep, J. Appl. Phys. , 91, 6872 (2002).

17 N. D. Mermin and H. Wagner, Phys. Rev. Lett. 17, 1133 (1966).

18 see for example R. Quartu and H.T. Diep, Phys. Rev. B 55, 2975 (1997).

19 R. A. Tahir-Kheli and D. Ter Haar, Phys. Rev. 127, 88 (1962).
20 N. N. Bogolyubov and S. V. Tyablikov, Doklady Akad. Nauk S.S.S.R. 126, 53 (1959) [translation: Soviet Phys.Doklady 4604 (1959)].

21 P. Fröbrich, P. J. Jensen and P. J. Kuntz, Eur. Phys. J B 13, 477 (2000) and references therein.

22 D. N. Zubarev, Usp. Fiz. Nauk 187, 71 (1960)[translation: Soviet Phys.-Uspekhi 3320 (1960)].

23 C. Santamaria, R. Quartu and H. T. Diep, J. Appl. Physics 84, 1953 (1998).

24 A. M. Ferrenberg and R. H. Swendsen, Phys. Rev. Let. 61, 2635 (1988).

25 A. M. Ferrenberg and R. H. Swendsen, Phys. Rev. Let. 63, 1195 (1989).

26 A. M. Ferrenberg and D. P. Landau, Phys. Rev. B 44, 5081 (1991).

27 P. Olsson and, Phys. Rev. B 71, 104423 (2005) and historical references cited therein.

28 E. H. Boubcheur and H. T. Diep, Phys. Rev. B 58, 5163 (1998).

29 G. Ramirez-Santiago and J.V.Jose, Phys. Rev. B 49, 9567 (1994).

30 S. Lee and K-C. Lee, Phys. Rev. B 49, 15184(1994).

31 J. Lee, J.M. Kosterlitz and E. Granato, Phys. Rev. B 43, 11531 (1991); E. Granato, J.M. Kosterlitz, J. Lee and M. P. Nightingale, Phys. Rev. Lett. 66, 1090 (1991).

32 M. P. Nightingale, E. Granato and J. M. Kosterlitz, Phys. Rev. B 52, 7402 (1995).

33 A. Zangwill, Physics at Surfaces, Cambridge University Press (1988).

34 Ultrathin Magnetic Structures, vol. I and II, J.A.C. Bland and B. Heinrich (editors), Springer-Verlag (1994). 